\title{
Preliminary simulations on the interpretation of cross-sectional Gaussian graphical models
}

\author{
Sacha Epskamp \\ University of Amsterdam: Department of Psychological Methods
}

This report describes two preliminary simulation studies that were included in earlier revisions of Epskamp, Waldorp, Mõttus, and Borsboom (2017). These will now be included in a future manuscript.

\section{Simulation Study 1: Cross-sectional Analysis and Repeated Measures}

We performed a simulation study to investigate the interpretability of GGMs obtained from cross-sectional data. We constructed two network structures: a within-subjects network and a between-subjects network. For the within-person network, we constructed a chain graph (as shown in Figure 1, panel (a)) in which each edge-weight was set to 0.25 and made negative with $50 \%$ probability. For the between-person network, we constructed the same structure but randomly rewired all the edges, such that a random graph was obtained (as shown in Figure 1, panel (b)). Next, the within-person network was scaled such that the within-person variance of all nodes was $0.1,0.2,0.5,1,2,5$ or 10 . The between-subjects variance was set to 1 . We estimated the network structure using glasso in combination with EBIC model selection (Foygel \& Drton, 2010), as implemented in the qgraph package (Epskamp, Cramer, Waldorp, Schmittmann, \& Borsboom, 2012; Epskamp \& Fried, 2017). We set the EBIC hyperparameter $\gamma$ to 0.25 .

Figure 2 shows the results, in which the estimated network was compared to the true within and between subjects networks as well as an unweighted network that contained an edge whenever there was an edge in either true network. We did not compute the correlation of edge weights with the unweighted graph as it contained no weights. The figure shows that in large differences between within-person and between-person variance the cross-sectional analysis converges to one of the two networks. When the between-subjects variance was relatively high the within-subjects network was not retrievable and vice versa. When the two variances were approximately equal, edges were detected that were in either of the networks (high sensitivity in the combined graph) and not many edges were detected that were not present in either network (high specificity). This indicates that detected edges in a cross-sectional network can be interpreted to likely represent an edge in the within-subjects network or in the between-subjects network. It should be noted that specificity did go down with increased sample size, as the true network does not necessarily contain zeros anymore. These false edges were usually estimated to be very weak. Finally, these results are based on a simulation study in which the within- and between-subject networks completely differed from each other. Preliminary results, such as the empirical samples in this paper, seem to 
suggest this is not the case in empirical data. We expect cross-sectional data analysis to perform better when the two network structures align.

In order to start disentangling within- and between-subjects variance, one needs an estimate of the person-specific mean. The simplest way to obtain this is by averaging two repeated measures. To this end, we repeated the simulation study above using the exact same setup, but now generated two independent responses per subject. Next, we centered the responses per subject to compute a within-subject network and used the sample means per subject to compute a between-subjects network. Figure 3 shows that the within-subject network could now be well retrieved, while the between-subject network could only be retrieved when the between-subject variance was large. This seems to suggest that now the estimated network structure can be more reliably interpreted to not be confounded by between-subjects variance. Care should still be taken in interpreting such results, as, for instance, the analysis assumes both no auto-regressions (these would greatly bias the estimated means per subject) and no individual differences in network structure (which one can not expect to obtain with only two repeated measures). We encourage future researchers to study cross-sectional and repeated measures analysis in more detail by performing more extensive simulation studies.

\section{References}

Epskamp, S., Cramer, A., Waldorp, L., Schmittmann, V. D., \& Borsboom, D. (2012). qgraph: Network visualizations of relationships in psychometric data. Journal of Statistical Software, 48(1), 1-18. doi: 10.18637/jss.v048.i04

Epskamp, S., \& Fried, E. I. (2017). A tutorial on estimating regularized psychological networks. arXiv preprint, arXiv:1607.01367.

Epskamp, S., Waldorp, L. J., Mõttus, R., \& Borsboom, D. (2017). Discovering psychological dynamics in time-series data. arXiv preprint, arXiv:1609.04156.

Foygel, R., \& Drton, M. (2010). Extended Bayesian information criteria for Gaussian graphical models. Advances in Neural Information Processing Systems, 23, 20202028. Retrieved from https://arxiv.org/abs/1011.6640 


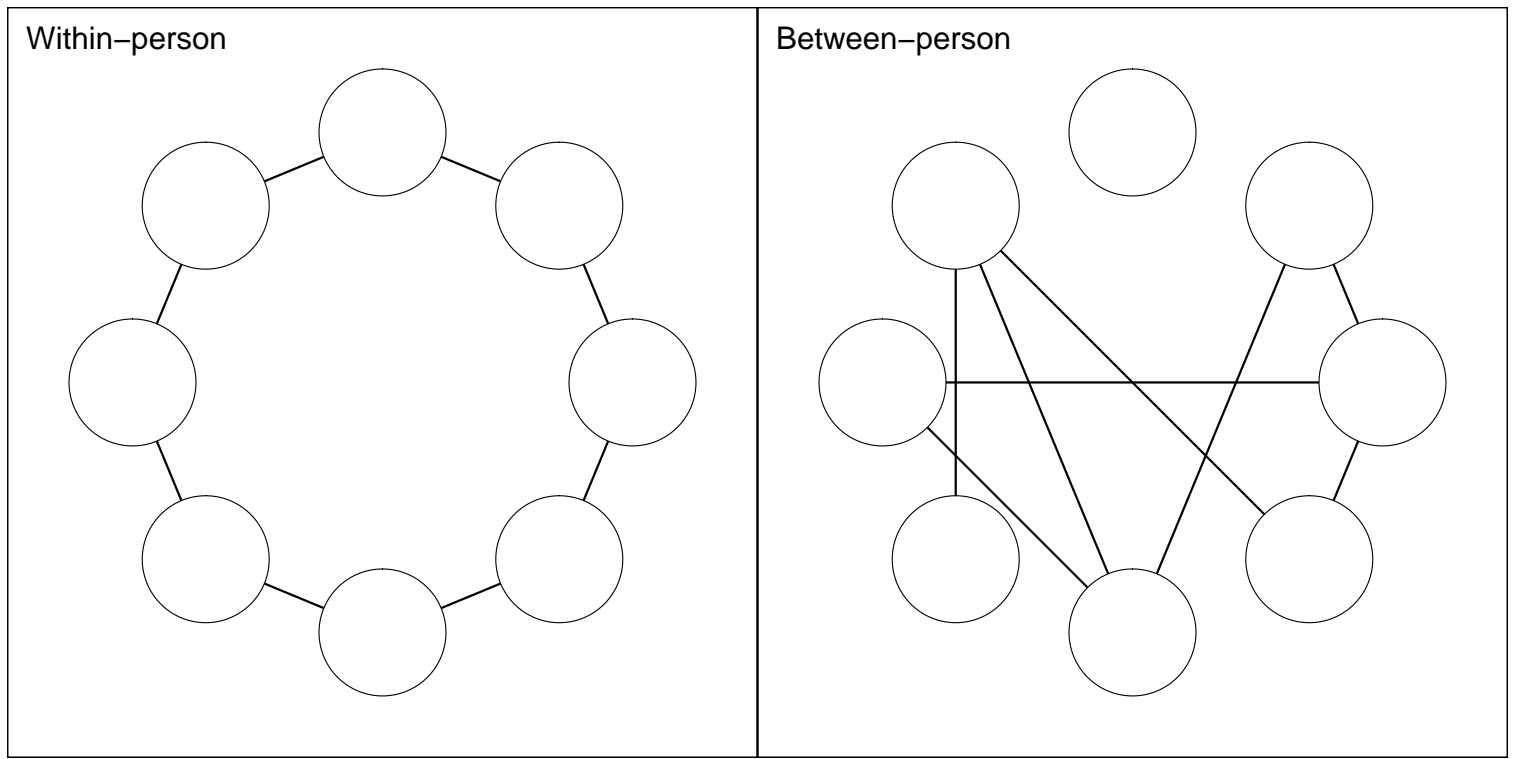

Figure 1. Example of simulated network models in the simulation study. The within-person network was simulated as a chain graph, and the between-subjects network was simulated as a random network with the same number of edges as the within-person network (a different random network was generated for each dataset). Non-diagonal elements were made negative with $50 \%$ probability. 
\# persons 白 100 官 250 它 500 追 1000
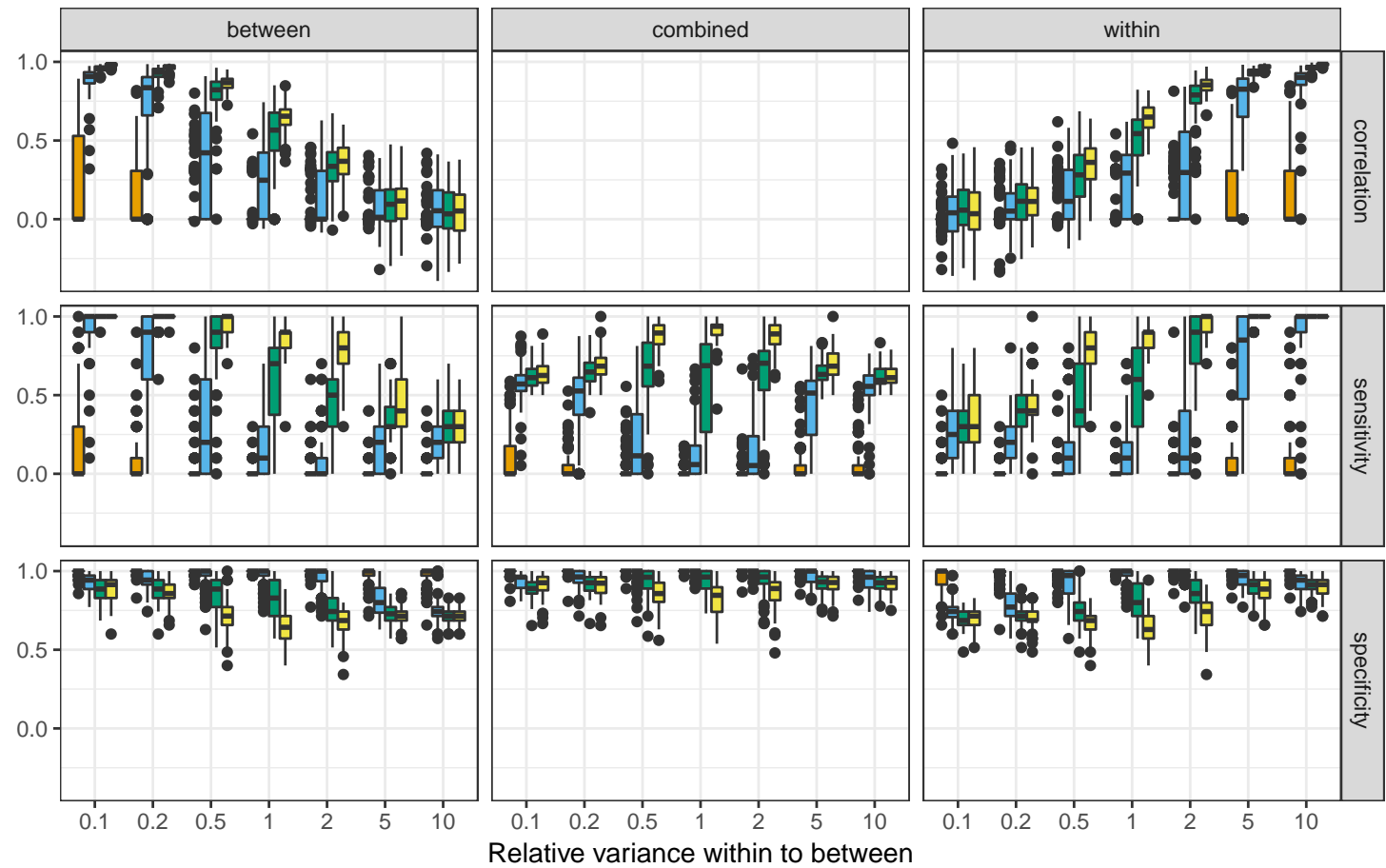

Figure 2. Simulations study showing the performance of cross-sectional analysis. Data were generated by summing scores generated from two distinct distributions: a chain graph GGM (within-person) and a random graph GGM (between-person). Left panels show performance compared to the between-subjects network and right panels show performance compared to the within-subjects network. The center panels show performance compared to an unweighted graph that contained an edge if there was an edge in the within-subjects network or the between-subjects network. 


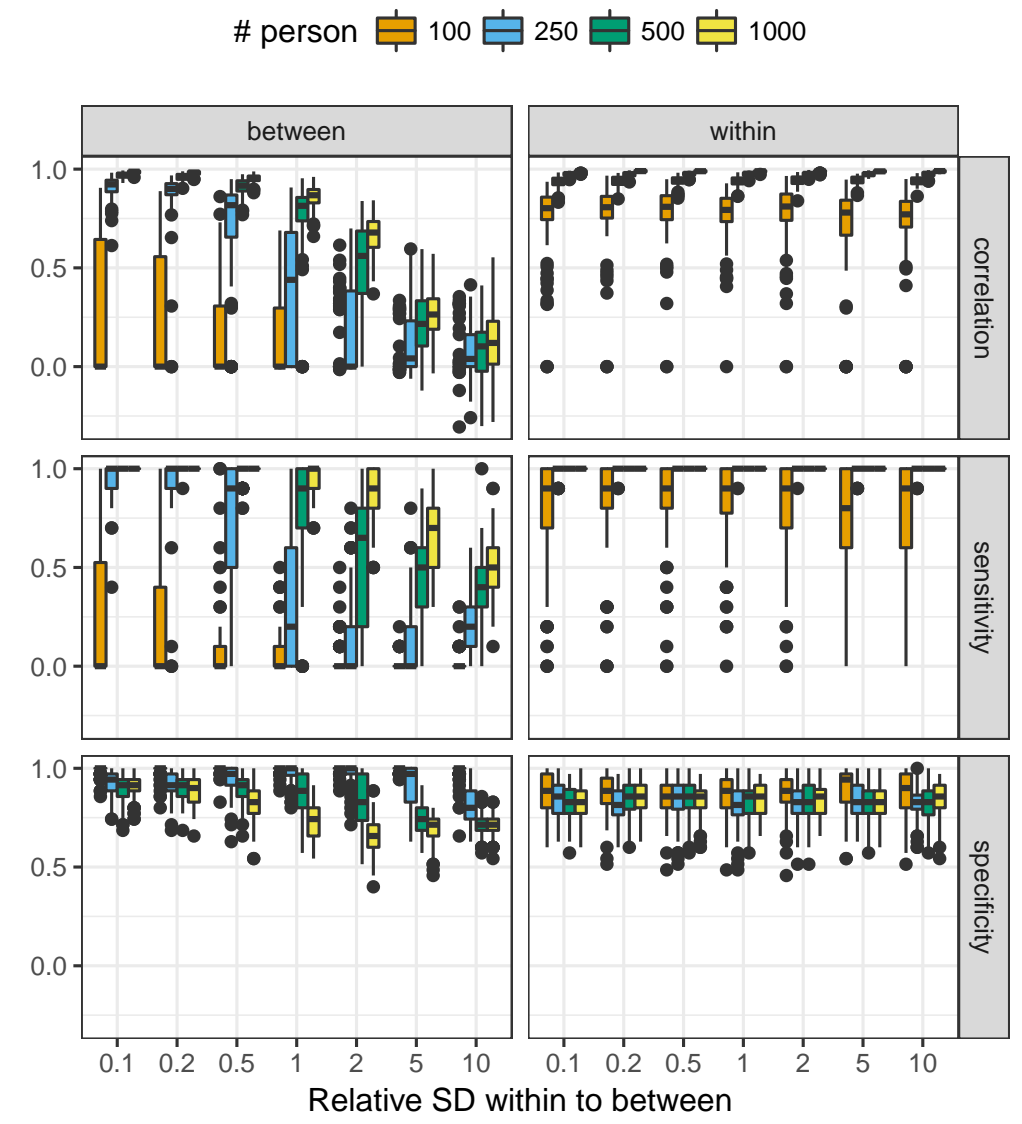

Figure 3. Simulations study showing the performance of cross-sectional analysis. Data were generated by summing scores generated from two distinct distributions: a chain graph GGM (within-person) and a random graph GGM (between-person). Left panels show performance compared to the between-subjects network and right panels show performance compared to the within-subjects network. The center panels show performance compared to an unweighted graph that contained an edge if there was an edge in the within-subjects network or in the between-subjects network. 